\title{
A Single-case Experiment for an Innovative Cognitive Behavioral Treatment of Auditory Hallucinations and Delusions in Schizophrenia
}

\author{
Eric Quintin \\ Northeast Kingdom Human Services (NKHS) \\ Vermont, USA \\ Claude Bélanger (Corresponding author) \\ University of Quebec in Montreal \\ $\&$ \\ McGill University and Douglas Institute of Mental Health \\ Montreal, Canada \\ E-mail: belanger.claude@uqam.ca \\ Valerie Lamontagne \\ University of Quebec in Montreal, Montreal, Canada
}

Received: November 16, 2011

Accepted: December 1, $2011 \quad$ Published: March 1, 2012

doi:10.5539/ijps.v4n1p114

URL: http://dx.doi.org/10.5539/ijps.v4n1p114

\begin{abstract}
This single-case experiment evaluates the impact of an innovative rational-emotive cognitive treatment focused on hallucination and delusions on quality of life, depression, anxiety, and insight in a female patient suffering from schizophrenia. The cognitive treatment developed for this experiment was directly inspired by the work of Ellis and Chadwick, Birchwood, and Trower. The rational-emotive cognitive approach used in the present study effectively reduced the patient's anxiety and depression, increased the patient's overall quality of life and insight. The gains were maintained at 3-month, 6-month, and 12-month follow-up. Future investigations into treatments for psychosis should focus on patients' insight regarding the origin of their hallucinations. Further, the link between negative and positive psychotic symptoms should be explored.
\end{abstract}

Keywords: Schizophrenia, Hallucinations, Delusions, Single-case study, Cognitive behavior therapy, Rational-emotive therapy

\section{Introduction}

Over the past decade, various cognitive-behavioral interventions and strategies have been successfully applied in clinical trials for affective disorders, anxiety disorders, and eating disorders (Gloaguen, Coattraux, Cucherat, \& Blackburn, 1998; Gould, Buckminster, Pollack, Otto, \& Yap, 1997; Whittal, Stewart, \& Gould, 1999). Led by researchers in the United Kingdom (Byrne, Birchwood, Trower, \& Meaden, 2006; Chadwick, Birchwood, \& Trower, 1996; Turkington, Kingdon, \& Chadwick, 2003; Turkington, Kingdon, \& Turner, 2002), interesting and innovative work in the field of cognitive psychology has resulted in the development and elaboration of cognitive therapy approaches to schizophrenia. The results of this recent body of research suggest that psychotic symptoms are not simply the product of physiological abnormalities in the brain. Rather, psychotic symptoms have meaning, and the majority of hallucinations and classic delusions observed in individuals with schizophrenia can be modified in therapy (Beck \& Rector, 2003; Byrne et al., 2006; Chadwick et al., 1996; Sensky, Turkington, \& Kingdon, 2000). Consequently, researchers in this area have been promoting the development and application of cognitive treatments for psychotic disorders such as schizophrenia. Such interventions often directly target specific symptoms such as delusions and hallucinations (Byrne et al., 2006; Chadwick et al., 1996; Turkington et al., 2002; Turkington et al., 2003). 
The rational-emotive cognitive model (Ellis, 1994; Dryden \& David, 2008; Dryden, David, \& Ellis, 2010) has been successfully applied to delusions in patients with schizophrenia and is now recognized as a compelling conceptualization of psychotic symptoms. In this $\mathrm{ABC}$ model, delusions are regarded as delusional interpretations or beliefs (B) about an activating event (A) and may be associated with consequences (C) such as distress and disturbances. The $\mathrm{ABC}$ model maintains that all delusions contain, at minimum, one explicit inferential thought and one implicit evaluative thought. Interventions based on this model have been demonstrated to effectively reduce erroneous beliefs and cognitive biases in psychotic patients (Bentall, 1996; Byrne et al., 2006; Chadwick et al., 1996). Further, this intervention model has been demonstrated to increase self-esteem and general functioning, and to decrease anxiety and depression in patients with schizophrenia (Wiersma, Jenner, Nienhuis, \& Willige, 2004).

Regarding auditory hallucinations, there is compelling evidence that the nature and strength of individuals' responses to such hallucinations are directly related to distorted interpretations of the voices; responses cannot be attributed to content because the same content heard in different voices evokes different reactions (Romme \& Escher, 1989; Tarrier, 1992). Chadwick and Birchwood (1994, 1995) identified four primary types of hallucination-related beliefs about voices: beliefs about voice identity, beliefs about voice purpose, beliefs about the power of the voice, and beliefs about compliance with the voice. Approximately $80 \%$ of voices heard by patients with schizophrenia are believed by the patients to be extraordinarily powerful (Chadwick et al., 1996). Patients' inability to influence the onset or cessation of the voices seems to contribute to the voice's perceived power. Moreover, voices often convey the impression of omniscience about the client's history, future, thoughts, emotions, and behavior. This impression often makes patients feel vulnerable, exposed, and confined; patients report a sense of powerlessness and helplessness (Gilbert, 1992). Empowering the patient and restoring his or her sense of personal control therefore constitutes one of the primary objectives of the cognitive therapy for psychotic symptoms proposed by Chadwick and Birchwood $(1994,1995)$.

Applications of Chadwick and Birchwood's cognitive approach to delusions and auditory hallucinations have yielded promising results; this approach has been successful in decreasing the distress and disturbance engendered by the positive symptoms of schizophrenia (Bentall, 1996; Chadwick \& Birchwood, 1994, 1995; Chadwick et al., 1996; Trower et al., 2004; Turkington et al., 2002). Numerous other direct and indirect benefits of the cognitive-behavioral approach have been reported, including the following: enhanced quality of life (Wiersma et al., 2004), decreased general anxiety and depression, improved self-esteem, empowerment and increased sense of personal control, and reduction in behavioral dysfunctions (Beck \& Rector, 2003; Pilling, Bebbington, \& Kuipers, 2002). Finally, significant reductions in compliance to hallucinatory voice commands following cognitive therapy for hallucinations have also been reported (Byrne et al., 2006).

The interaction between delusions and hallucinations in individuals with schizophrenia warrants consideration in treatment. Delusional thinking does not seem to be constantly present; there is growing evidence that some delusions are triggered by activating events (e.g., perceptual abnormalities), whereas others reflect individual psychological motivations (Garety, 1991). In some cases, hallucinations may trigger delusional thinking. The development of treatments that target hallucinations and delusions simultaneously seems to be a valuable next step.

\subsection{Research Objectives}

This single-case study evaluated the impact of a new rational-emotive cognitive treatment for schizophrenia. The treatment integrated components from the rational-emotive model (Ellis, 1994; Dryden et al., 2010), as applied to delusions (Bentall, 1996), and from cognitive therapy for hallucinations, as applied by Chadwick and colleagues (1996). More specifically, we examined the impact of this new intervention on the patient's quality of life, as well as her level of anxiety and depression, and her insight into the origin of her auditory hallucinations.

\section{Method}

A classic single-case A-B-A or reversal design was used. Each pre-established variable was assessed and quantified with two measurements at baseline, two weeks prior to the introduction of the treatment. Variables were measured at weeks 9 and 16 during treatment, and again at the end of treatment. Following treatment termination, follow-up measures were completed at three-month, six-month, and twelve-month follow-up. All psychotropic medications taken by the patient during treatment were systematically monitored and recorded in order to control for this variable and identify adjustments to medication that could impact the patient's behavior or the other outcome variables.

Quantitative statistical analysis was conducted with the data from each of the four WHOQOL domains and the 
two general quality of life questions. A single-case time series scoring algorithm (C statistic) was used to analyze the data and identify any statistically significant effect of therapy on quality of life. The same algorithm was used for quantitative analysis of the results of the depression, anxiety, and insight measures, in order to determine the impact of therapy on these variables. The C statistic was calculated according to Jones' (2003) method. The time series $\mathrm{C}$ statistic is the most convenient form of statistical analysis for case study data because it makes few assumptions about the form of the data and its standard error is a function of the number of available observations. The criterion for significance and acceptance of the experimental hypothesis for all variables was established at $p=.05$.

\subsection{Participant}

The patient provided written informed consent, a copy of which was added to her file. The patient was a single 37-year-old American woman, diagnosed with schizophrenia at age 24. The diagnosis was established by a licensed psychiatrist and corroborated by an evaluation by the first author, using DSM-IV-TR (APA, 2000) diagnostic criteria. At the time of the study, the patient was living with her parents. She had been suffering from auditory hallucinations and delusional thinking for over 10 years, in spite of many trials of first and second generation antipsychotic medications. No significant effect of the medications on positive symptoms was reported. The patient was monitored by a psychiatrist during the study, and was treated with the following medications during the treatment and the follow-up period: a major tranquilizer (Clozaril $500 \mathrm{mg} / \mathrm{HS}$ ), two antidepressants (Ziprasidone $120 \mathrm{mg} / \mathrm{BID}$, Escitalopram $20 \mathrm{mg} / \mathrm{DIE}$ ), and an anticonvulsant (Topiramate 100 $\mathrm{mg} / \mathrm{am}, 150 \mathrm{mg} / \mathrm{pm})$. The patient had not previously received psychotherapy or cognitive-behavioral therapy. She was given a two-hour briefing about the processes and objectives of the current study, during which all of her questions about the study and the therapy were answered.

The patient's clinical presentation was typical of schizophrenia with paranoid features. The first episode of hallucination and delusional thinking occurred subsequent to a divorce at the age of 24 years. Prior to the implementation of cognitive treatment, the patient manifested unambiguous delusional thinking and auditory hallucinations that were resistant to medication. Prior to the start of therapy, she had been having 5 to 10 discrete hallucinatory episodes per day. The hallucinations were primarily auditory; they took the form of persecutory voices that commented on the patient's physical appearance and gave voice commands or warnings. The client repeatedly mentioned that the voices seemed to know everything about her; she felt that resistance to the voices was futile (i.e., she felt helpless and hopeless), given their perceived power and the possible negative consequences of non-compliance.

\subsection{Measures}

World Health Organization Quality of Life Schedule: The 26-item American self-report version of the WHOQOL-BREF (Bonomi \& Patrick, 1997) was used. This measure includes two items about quality of life and satisfaction with general health; the remaining 24 items are grouped into four domains (physical, psychological, social relationships, and environment), each of which is respectively measured on a five-point scale. The total raw score is the sum of the 26 items; the maximum total raw score is 130 . The 26 -item version of the measure was normalized and validated in the United States (Bonomi \& Patrick, 1997) and is stable $(r>.80$ on all domains) and reliable ( $r>.80$ on all domains). Convergent validity and discriminant validity were established by authors of the WHOQOL-BREF using ANOVA analysis with controls for extraneous factors (age and gender). Finally, validation of the questionnaire was performed using factor analyses that were conducted to demonstrate correlations within related domains.

The Burns Depression Checklist (BDC) is a 15-item psychometric instrument (Burns, 1999) that uses a 4-point Likert scale (0-3) to evaluate the intensity of common depressive symptoms over the past two weeks. Scores indicate one of five levels of depression, from minimal or no depression (0 to 4) to severe depression (31 to 45). Correlation in previous studies between the BDC and the Beck Depression Inventory (BDI; Beck, 1976) is strong; normalization for the BDC was based on scores on corresponding BDI items. Both The BDI and BDC have good internal consistency, strong content validity, excellent concurrent validity, and well established discriminative validity in previous clinical studies. Thus given their well-established empirical support, both are seen as valid, self-report measures of depression.

The Burns Anxiety Inventory (BAI) is a 33-item psychometric instrument (Burns, 1999) that uses a 4-point Likert scale (0-3) to evaluate the intensity of common anxiety symptoms. Scores indicate one of six levels of anxiety, from minimal or no anxiety (0 to 4) to extreme anxiety or panic (51 to 99 ). Results obtained in a previous study done with 498 outpatients seeking treatment for mood disorders suggested excellent reliability 
and internal consistency $(\alpha=.94)$; the test-retest reliability coefficient was $.53(\mathrm{p}<.0001)$ over a 12 -week period. (Burns, 1999).

Insight into the origin of auditory hallucinations is assessed by a direct measure of the patient's insight regarding her hallucinations. The measure required the patient to indicate her perception of the voices' origin on a scale from 0 to 100 on which 0 indicates complete certainty that the voices are external and 100 indicates complete certainty that the voices are internal and represent symptoms of schizophrenia.

\subsection{Treatment}

A new cognitive treatment was implemented. The treatment integrated components of the cognitive therapy for hallucinations developed by Chadwick and colleagues (1996), and components of Ellis' rational-emotive therapy (Ellis, 1994; Dryden \& David, 2008; Dryden et al., 2010) as applied to delusions by Bentall (1996). See Table 1 for a summary of the content of the therapy sessions.

\section{Insert Table 1 Here}

Inclusion of relatives in the treatment procedure was desirable; psychoeducation for the patient and her family was implemented at the beginning of therapy and integrated as required during therapy sessions. Various coping techniques (e.g., recording, self-monitoring, incompatible behavior, sensory deception, counter-stimulation) were used as needed during therapy; the techniques were designed to allow the patient to maintain control over potentially overwhelming positive symptoms, and to prevent potential psychotic breakdown or relapse. Homework assignments requiring behavioral testing were specifically tailored and structured to increase insight and decrease beliefs ascribing power and omnipotence to the voices. A treatment evaluation measure was used to verify and confirm that treatment objectives were achieved according to protocol (Trower et al., 2004).

\section{Results}

Data from the different measures were obtained at eight different points during intervention (including pre-treatment and follow-up). Results are presented in Table 2.

\section{Insert Table 2 Here}

\subsection{Quality of Life}

The results to questions one $(M=3.38, S D=0.92, \mathrm{C}=0.83, z=2.69$ and $p=.004)$ and two $(M=3.75, S D=$ $0.46, \mathrm{C}=0.67, z=2.16$ and $p=.015)$ on the WHOQOL-BREF indicated a significant improvement in global quality of life.

The results on the four domains of the WHOQOL-BREF were interesting. Although the patient's score on physical quality of life (domain 1) was already in the acceptable range at baseline, significant improvement over the course of therapy was observed and maintained at follow-up. The improvement in quality of life in this domain was particularly significant $(M=87.38, S D=11.88, \mathrm{C}=0.80, z=2.59$ and $p=.005)$.

The patient's psychological quality of life (domain 2) was 42 at baseline, and increased to 67 at post-treatment. The observed effect was maintained at all three follow-up evaluations $(58,63$, and 63 , respectively). These scores approach the norm for healthy individuals; the gains observed over the course of therapy were statistically significant $(M=55.88, S D=8.94, \mathrm{C}=.75, z=2.42$ and $p=.008)$.

Results on the social quality of life domain (domain 3) were non-significant $(M=53, S D=5.95, \mathrm{C}=.03, z=.1$ and $p=.54$ ). That is, the results for this domain did not permit the rejection of the null hypothesis; it appears that the treatment had no impact on the patient's social quality of life.

Similarly, the results on the environmental quality of life domain indicated no significant effect of treatment ( $M$ $=86.38, S D=9.83, \mathrm{C}=0.28, z=0.90$ and $p=.19$ ). However, the patient's score at baseline (78) was already above the norm for healthy individuals and remained stable for the duration of therapy.

\subsection{Anxiety}

The patient's anxiety at baseline (pre-tests 1 and 2) was in the severe range (31 to 50). Her anxiety levels decreased gradually and consistently during the intervention period, and were in the minimal range (0 to 4$)$ at post-test. These statistically significant gains were maintained at six-month, nine-month, and twelve-month follow-up, respectively. Time series analyses using $\mathrm{C}$ statistic calculations $(M=15.13, S D=15.01, \mathrm{C}=0.78$ and $z=2.54)$ revealed that the probability of obtaining these results by chance was negligible $(p=.006)$.

\subsection{Depression}

The patient's level of depression decreased gradually and consistently over the course of intervention. The 
observed significant decrease was maintained following therapy; the patient's depression was in the minimal range (0-4) at twelve-month follow-up. This result suggests not only that the gains achieved during therapy were maintained at follow-up, but that the patient subsequently made additional gains. Time series analysis revealed a high level of significance $(M=10.50, S D=8.00, \mathrm{C}=0.82$ and $z=2.65)$ and a critical probability $(p=.004)$ that permitted the rejection of the null hypothesis.

\subsection{Insight}

The data collected during the baseline period revealed that the client was unaware (insight $=10$ ) that the auditory hallucinations and voices were internally generated. Significant gains in awareness over the course of intervention were observed and were maintained at follow-up. Time series analysis revealed statistically significant results $(M=46.25, S D=24.46, \mathrm{C}=0.81, z=2.62$ and $p=.004)$ that permitted the rejection of the null hypothesis.

\section{Discussion}

The results presented in this single-case experiment indicate that the innovative rational-emotive cognitive treatment (Ellis, 1994; Dryden et al., 2010) administered to the patient increased her overall quality of life (Bonomi \& Patrick, 1997). In particular, the patient's psychological and physical quality of life improved over the course of therapy, with evidence of increased or maintained gains six to twelve months post-treatment. The results on all of the quality of life domains were similar to those reported by Wiersma and colleagues (2004). The therapy did not significantly impact the patient's environmental quality of life; however, it is worth noting that the patient's score on this domain was elevated compared to the WHOQOL group norms prior to the beginning of therapy. The patient's good premorbid environmental quality of life may have been a facilitator or catalyst for the positive impact of therapy (Wiersma et al., 2004). The patient's social quality of life did not fluctuate significantly over the course of therapy. The lack of observed change in the patient's environmental and social quality of life was not unexpected; the treatment was developed and structured to focus primarily on elements related to her behavioral and psychological quality of life (Wiersma et al., 2004).

The cognitive treatment implemented in the study presented here effectively decreased anxiety symptoms (Gould et al., 1997); whereas significant anguish and distress were reported prior to therapy, post-treatment anxiety was minimal to absent. This result was corroborated by clinical observations of the client's behavior. The patient's lack of confidence in her social skills and corresponding low self-esteem were only partially addressed during therapy; these issues should be the focus of secondary behavioral intervention designed expressly to address her lack of social skills (Pilling et al., 2002). Given that the patient's anxiety has decreased considerably, complementary therapy could enable further significant gains in social quality of life (Bonomi \& Patrick, 1997). Therapy also had a significant impact on the patient's symptoms of depression (Gloaguen et al., 1998); her symptoms decreased steadily over the course of treatment, and effects were maintained at six-month, nine-month, and twelve-month follow-up. Improvement in the patient's mood may have been a catalyst for the other therapeutic gains observed over the course of therapy. Alleviation of depressive symptoms may have allowed her to be more conscientious, present, and energetic during therapy, facilitating the therapeutic process and enhancing subsequent gains.

The therapy had a significant and enduring impact on the client's insight relative to her hallucinations and to the origin of her delusions (Beck et al., 2003). Initial gains were discernable after the first few therapy sessions; significant improvements were observed after the mid-point of therapy, with gains maintained at follow-up.

Although the patient was still not entirely convinced of the internal provenance of the auditory hallucinations and voices following therapy (Chadwick \& Birchwood, 1994, 1995), her evident increase in insight was central to her observed progress in the cognitive elements of therapy. The majority of the therapeutic concepts and fundamental precepts in the treatment required the patient's acknowledgement that her hallucinations and delusions were the product of her mind and did not originate from an external stimulus (Garety, 1991). Her gains in insight in the first portion of therapy provided the necessary platform for subsequent cognitive restructuring, and permitted the patient and the therapist to effectively challenge the patient's distortions in her cognitions. At the end of treatment and at follow-up, her acquired resistance to hallucinations, delusions, and, in particular, voice commands enabled the patient to enjoy activities that had previously been problematic or inaccessible for her.

One caveat regarding the future use of this type of therapy must be issued: therapists must be careful when they challenge delusions and distorted cognitions of client's with schizophrenia. This population tends to be quite resistant to such challenges; cognitive restructuring must be implemented with the utmost care in order to avoid 
emotional distress that could threaten or obstruct the therapeutic process.

\subsection{Limitations and Future Directions}

There are limitations to the present study. First, since the research was based on a single case, the generalization of the findings is limited; caution must be used in extrapolating the results to all individuals with schizophrenia. However, since external validity is achieved through replication, both in single-case and traditional group designs, it could be argued that multiple single-case replications could have greater scientific value than would one replication with a large sample. Second, since this research was conducted in a natural environment rather than in a laboratory setting, there were added difficulties in controlling external and potentially confounding variables. Nonetheless, common external confounds were excluded or controlled to the degree possible, given the natural setting of the experiment. Third, the use of the brief version of the WHOQOL (Bonomi \& Patrick, 1997), rather than of the original version, constitutes a limitation in that the brief measure is resistant to minor variations in response patterns. For example, in the section about social quality of life, even though no significant change was observed in this case, fewer changes in response are required to make the score fluctuate than would be with the original version of the measure. For example, the use of the brief version of the measure could account for the observed change in score from the first follow-up to the third follow-up. Another limitation of the study might be related to therapeutic alliance, a factor that was not measured but that could have influenced symptom improvement (for a review see Martin, Garske, \& David, 2000; Frank \& Gunderson, 1990,). Finally, in order to reduce the administration time and maximize compliance, insight was assessed with a unidimensional measure. Results should be replicated using a validated comprehensive measure of different insight aspects like the Scale for the Assessment of Unawareness of Mental Disorder (SUMD, developed by Amador et al. 1993).

The major remaining questions in this area concern a) the relationship between negative and positive psychotic symptoms, and b) clinical interventions with the potential to provide further symptom relief for individuals suffering from schizophrenia and other psychotic disorders. Environmental and social quality of life for individuals with schizophrenia remains a further question of primary interest; there are still many options for clinical approaches designed specifically to address these elements. General cognitive and behavioral approaches, such as social skills and assertiveness training, communication and problem-solving skills, and self-esteem oriented therapy, may prove to be interesting avenues. The inclusion of adjunct group therapy and family therapy to the CBT approach described here could enhance patients' social and environmental quality of life. Finally, because insight appears to be a primary factor in clinical progress in schizophrenia clients, further research about these patients' insight into the origin of hallucinations and delusions is warranted.

\section{References}

American Psychiatric Association. (2000). Diagnostic and statistical manual of mental disorders IV-R. (4th ed.). Washington, DC: American Psychiatric Association.

Amador, X. F., Flaum, M., Andreasen, N. C., Strauss, D. H., Yale S. A., \& Clark S. C. et al. (1994). Awareness of illness in schizophrenia and schizoaffective and mood disorders. Archives of General Psychiatry, 51 (10), 826-836. http://dx.doi.org/10.1001/archpsyc.1994.03950100074007

Beck, A. T. (1976). Cognitive therapy and the emotional disorder. New York: International Universities Press.

Beck, A. T., \& Rector, N. A. (2003). A cognitive model of hallucinations. Cognitive Therapy and Research, 27, 19-52. http://dx.doi.org/10.1023/A:1022534613005

Bentall, R. P. (1996). From cognitive studies of psychosis to cognitive-behavior therapy for psychotic symptoms. In G. Haddock, \& P. D. Slade (Eds.). Cognitive-behavioral interventions with psychotic disorders (pp. 3-27). New York: Routledge.

Bonomi, A. E., \& Patrick, D. L. (1997). User's manual and interpretation guide for the United States version of the World Health Organization Quality of Life (WHOQOL) instrument. Seattle,WA: U.S. WHOQOL Center.

Burns, D. D. (1999). The Burns Depression Checklist (BDC) and the Burns Anxiety Inventory (BAI). In The feeling good handbook (Revised edition). New York: Plume.

Byrne, S., Birchwood, M., Trower, P., \& Meaden, A. (2006). A casebook of cognitive behavior therapy for command hallucinations: A social rank theory approach. New York: Routledge.

Chadwick, P., \& Birchwood, M. (1994). The omnipotence of voices: A cognitive approach to auditory hallucinations. The British Journal of Psychiatry, 164, 190-201. http://dx.doi.org/10.1192/bjp.164.2.190

Chadwick, P., \& Birchwood, M. (1995). The omnipotence of voices II: The beliefs about voices questionnaire. 
British Journal of Psychiatry, 166, 11-19. http://dx.doi.org/10.1192/bjp.166.6.773

Chadwick, P., Birchwood, M., \& Trower, P. (1996). Cognitive therapy for delusions, voices and paranoia. Chichester: Wiley.

Dryden, W., \& David, D. (2008). Rational emotive behavior therapy: Current status. Journal of Cognitive Psychotherapy. 22 (3),195-209. http://dx.doi.org/10.1891/0889-8391.22.3.195

Dryden, W., David, D., \& Ellis, A. (2010). Rational emotive behavior therapy. Handbook of cognitive-behavioral therapies. (3rd ed.). New York, NY, US: Guilford Press.

Ellis, A. (1994). Reason and emotion in psychotherapy. New York: Lyle Stuart.

Frank, A. F., \& Gunderson, J. G. (1990). The role of the therapeutic alliance in the treatment of schizophrenia. Archives of General Psychiatry, 47 (3), 228-236.

Garety, P. A. (1991). Reasoning and delusions. British Journal of Psychiatry, 159, 14-18.

Gilbert, P. (1992). Depression: The evolution of powerlessness. Hove: Lawrence Erlbaum.

Gloaguen, V., Coattraux, J., Cucherat, M., \& Blackburn, I. M. (1998). A meta-analysis of the effects of cognitive therapy in depressed patients. Journal of Affective Disorders, 49 (1), 59-72. http://dx.doi.org/10.1016/S0165-0327(97)00199-7

Gould, R. A., Buckminster, S., Pollack, M. H., Otto, M. W., \& Yap, L. (1997). Cognitive-behavioral and pharmacological treatment for social phobia: A meta-analysis. Clinical Psychology, Science and Practice, 4 (4), 291-306. http://dx.doi.org/10.1111/j.1468-2850.1997.tb00123.x

Jones, W. P. (2003). Single-case time series with Bayesian analysis: A practitioner's guide. Measurement and evaluation in counseling and development, 36, 28-39.

Martin, D. J., Garske, J. P., \& David, M. K. (2000). Relation of the therapeutic alliance with outcome and other variables: a meta-analytic review. Journal of Consulting and Clinical Psychology, 68, 438-450. http://dx.doi.org/10.1037//0022-006X.68.3.438

Pilling, S., Bebbington, P., \& Kuipers, E. (2002). Psychological treatments in schizophrenia I: Meta-analysis of family intervention and CBT. Psychological Medicine, 32, 763-782. http://dx.doi.org/10.1017/S0033291702005895

Romme, M. A., \& Escher, S. (1989). Hearing voices. Schizophrenia bulletin, 15, 209-216.

Sensky, T., Turkington, D., \& Kingdon, D. (2000). A randomized controlled trial of cognitive-behavioral therapy for persistent symptoms in schizophrenia resistant to medication. Archives of General Psychiatry, 57, 165 -172. http://dx.doi.org/10.1001/archpsyc.57.2.165

Tarrier, N. (1992). Management and modification of residual positive psychotic symptoms. In M. Birchwood, \& N. Tarrier (Eds.). Innovations in the psychological management of schizophrenia (pp. 147-169). Chichester: Wiley.

Trower, P., Birchwood, M., Meaden, A., Byrne, S., Nelson, A., \& Ross, K. (2004). Cognitive therapy for command hallucinations: Randomized controlled trial. British Journal of Psychiatry, 184, 312-320. http://dx.doi.org/10.1192/bjp.184.4.312

Turkington, D., Kingdon, D., \& Chadwick, P. (2003). Cognitive-behavioral therapy for schizophrenia: Filling the therapeutic vacuum. The British Journal of Psychiatry, 183, 98-99. http://dx.doi.org/10.1192/bjp.183.2.98

Turkington, D., Kingdon, D., \& Turner, T. (2002). Effectiveness of a brief cognitive-behavioral therapy intervention in the treatment of schizophrenia. British Journal of Psychiatry, 180, 523-527. http://dx.doi.org/10.1192/bjp.180.6.523

Whittal, M. L., Stewart A. W., \& Gould, R. A. (1999). Bulimia nervosa: A meta-analysis of psychosocial and $\begin{array}{llllll}\text { pharmacological treatments. } & \text { Behavior } & \text { Therapy, } & 30 & \text { (1), }\end{array}$ http://dx.doi.org/10.1016/S0005-7894(99)80049-5

Wiersma, D., Jenner, J. A., Nienhuis, F. J., \& Willige G. (2004). Hallucination focused integrative treatment improves quality of life in schizophrenia patients. Acta Psychiatrica Scandinavica, 109, 194-201. http://dx.doi.org/10.1046/j.0001-690X.2003.00237.x 
Table 1. Summary of rational-emotive cognitive therapy session content

\begin{tabular}{|c|l|}
\hline Session \# & Summary of content \\
\hline$(1-2)$ & $\begin{array}{l}\text { Establish rapport, discuss current problems, past history, and the patient's situation. } \\
\text { Introduce the ABC model and the relevance of the model, collaboratively establish target goals } \\
\text { and problems. Targets must have an activating event (A) and a consequence (C), and may include } \\
\text { a related belief (B). Identify emerging themes in specific cognitive distortions. } \\
\text { Assess activating events and/or consequences, evaluate the importance of problems by severity } \\
\text { and type. } \\
\text { Assess remaining activating events (A) or consequences (C). Clarify and identify specific triggers. } \\
\text { Assist the patient in discriminating between subjective judgment and objective facts, to facilitate } \\
\text { future challenges to distorted representations. }\end{array}$ \\
$(9-8)$ & $\begin{array}{l}\text { Connect the activating events (A) to consequences (C) and ensure that you understand the } \\
\text { patient's concerns. Communicate understanding of the dynamic to the client. } \\
\text { Use thought chaining to assess beliefs (B) without directly challenging them. Help the patient } \\
\text { recognize that activating events (A) are not responsible for emotional reactions or behavior (C). } \\
\text { Guide the patient's evolution from an A-C perspective to an A-B-C perspective. }\end{array}$ \\
$(13-12)$ & $\begin{array}{l}\text { Use a cognitive developmental perspective to make the connection between beliefs (B) about } \\
\text { voices and consequences (C). Identify specific vulnerabilities. } \\
\text { Restate or adjust patient's goals and consider options. Compare patient's previous A-C } \\
\text { formulation and facilitate the development of a new A-B-C perspective. Present the question: In } \\
\text { your opinion, what would have to change in order to resolve your problems? } \\
\text { Challenge beliefs more directly, starting with peripheral beliefs and progressing to more central } \\
\text { ones. Use cognitive restructuring, empirical testing, Socratic questioning, belief replacement, and } \\
\text { evidence review to moderate or modify beliefs. Challenge and test inferences and evaluations } \\
\text { about voices and auditory hallucinations. } \\
\text { Feedback, assessment of goal attainment, patient questions, and remaining issues. }\end{array}$ \\
$(15-16)$ & $\begin{array}{l}\text { (20) } \\
\text { (17-19) }\end{array}$ \\
\hline
\end{tabular}

Table 2. Psychometric results

\begin{tabular}{|l|l|l|l|l|l|l|l|l|}
\hline \multicolumn{1}{|c|}{ Psychometric test } & \multicolumn{1}{|c|}{$\begin{array}{c}\text { Pre1 } \\
(1)\end{array}$} & $\begin{array}{c}\text { Pre2 } \\
(2)\end{array}$ & $\begin{array}{c}\text { Dur1 } \\
(3)\end{array}$ & $\begin{array}{c}\text { Dur2 } \\
(4)\end{array}$ & $\begin{array}{c}\text { Post } \\
(5)\end{array}$ & $\begin{array}{c}\text { Fol1 } \\
(6)\end{array}$ & $\begin{array}{c}\text { Fol2 } \\
(7)\end{array}$ & $\begin{array}{c}\text { Fol3 } \\
(8)\end{array}$ \\
\hline WHOQOL-BREF Q1 General quality (1-5) & 2 & 2 & 3 & 4 & 4 & 4 & 4 & 4 \\
\hline WHOQOL-BREF Q2 General health (1-5) & 3 & 3 & 4 & 4 & 4 & 4 & 4 & 4 \\
\hline WHOQOL-BREF D1 Physical (0-100) & 71 & 75 & 79 & 82 & 100 & 96 & 96 & 100 \\
\hline WHOQOL-BREF D2 Psychological (0-100) & 42 & 46 & 50 & 58 & 67 & 58 & 63 & 63 \\
\hline WHOQOL-BREF D3 Social (0-100) & 58 & 58 & 50 & 50 & 58 & 42 & 50 & 58 \\
\hline WHOQOL-BREF D4 Environment (0-100) & 78 & 78 & 78 & 88 & 100 & 78 & 91 & 100 \\
\hline Burns Anxiety Inventory (0-99) & 41 & 37 & 12 & 8 & 4 & 4 & 6 & 9 \\
\hline Burns Depression Checklist (0-45) & 23 & 23 & 11 & 7 & 6 & 5 & 5 & 4 \\
\hline Insight (0-100) & 10 & 10 & 40 & 50 & 70 & 60 & 60 & 70 \\
\hline
\end{tabular}

Note. Pre $=$ pre-test (two measurements at baseline, one and two weeks prior to introduction of treatment)

Dur $=$ measures during the course of therapy (measured at weeks 9 and 16 during treatment)

Fol $=$ Follow-up measures (completed at three-month, six-month, and twelve-month follow-up) 